\title{
The Soon to Be Immortal Talks to Himself Randy Phillis
}

I have given it all away except hope: the house, the clothes, the furniture, the mementos, the incense. It's a long journey. I'm pointing toward heaven and they're waiting.

I'm ready and so are they.

I'm as light as pine needles:

After three years of only the needlesno rice, no wheat, no quail, no fish-I am very light. As light as I can be.

And here I stand, the rocks of this spur pricking my feet for the last time. Below me all the faces who come to see, laughing, believing, above swirling blue like the robes I'll wear when I arrive like a swan.

Below me, only the faces. 